\section{A comment on "The effects of framing ratio and oblique length on Ponzo illusion magnitude"}

\author{
ALEXANDER W. PRESSEY \\ University of Manitoba \\ Winnipeg, Manitoba, Canada
}

In their well-conceived experiment on the Ponzo illusion, Jordan and Randall (1987) argue that a revised version of assimilation theory proposed by Pressey and Wilson (1980) is capable of explaining their results. They describe a central attentional field in which stimuli are assimilated and a surrounding contrast field in which differences between stimuli are enhanced. They go on to show how several complicated inverted-U trends are explained on the basis of such a bidirectional attentive field.

However, the model they describe is not the one proposed by Pressey and Wilson (1980). Rather, it appears to be a meld of constructs from assimilation theory and constructs from "dual-glance"' theories, such as, for example, the pool-and-store model (Coren \& Girgus, 1978). The difference is that Pressey and Wilson contend that contrast is mediated not by attention deployment, but through the "interactive field" that reflects spatial contour interaction effects. Indeed, they likened the interactive field to receptive fields found by single-cell recordings in which a central excitatory (or inhibitory) field is surrounded by an outer inhibitory (or excitatory) ring. ${ }^{1}$

A second point of disagreement between the published version of assimiliation theory and the Jordan-Randall view is in the definition of the attentional field. They claim that, according to the theory, the radius of the attentional field is a constant ratio of test line length. In fact, the theory states that the radius of the field is a constant ratio of target size. But the target includes both the test line and the comparison line. One of the unique aspects of assimilation theory is that it includes the measuring device as an integral part of the theory. It is because of this inclusion that it has been able to explain not only many of the main effects of the Ponzo illusion, but also reversed effects that can be induced under special conditions (Pressey, Butchard, \& Scrivner, 1971).

If the appropriate definition of the attentional field ${ }^{2}$ is applied to the Jordan-Randall target, then the minimum radius would be about $135 \mathrm{~mm}$ and the maximum field would have a radius of approximately $270 \mathrm{~mm}$. This means that the nonmonotonic effect of oblique length would be found only in the condition that had the largest ratio of contextual to test line length. But this derivation

Research on assimilation theory was supported by the Natural Sciences and Engineering Research Council of Canada and by the Manitoba Mental Health Research Foundation. Requests for reprints should be sent to Alexander Pressey, St. John's College, University of Manitoba, Winnipeg, MB R3T 2M5, Canada. is clearly not what the results show, and thus it does not appear that the Jordon-Randall variant can be successfully coupled with Pressey's assimilation theory to explain the data obtained by Jordan and Randall.

Of course, it may well be that the operational definition of an attentional field provided by assimilation theory holds only for cases in which the comparison line is fairly close to the standard line. In such a case, both standard and comparison lines would be apprehended as a single whole and a single circle might represent the attentional field quite well. If the comparison line is placed farther and farther away from the standard, however, the shape of the field might become elliptical, then "dumbbell" shaped, and then differentiated into two separate circular fields-one surrounding the standard line and one surrounding the comparison line. This last condition would satisfy the Jordan-Randall definition of an attentional field ${ }^{3}$ and allow their explanation of the Ponzo data to be viable.

It should be noted, at this point, that if contrast is mediated through the contour interaction component (the interactive field) as Pressey and Wilson (1980) suggest, the details of that process would be exactly like those specified by Jordan and Randall. The latter tie their attentional field to the standard line very much like the former tie the interactive field to the standard line. Thus, the logic for deriving the empirical functions would be identical.

A third point of disagreement stems from Jordan and Randall's claim that 'Pressey and Wilson's (1980) revision of the theory does not account for age-related trends in the Ponzo illusion"' (p. 439). The difficulty with this contention is that it implies that there is an unequivocally established relationship between age and Ponzo illusion that must be explained, whereas in fact no such relationship has been established. For example, Leibowitz and his colleagues (Farquhar \& Leibowitz, 1971; Leibowitz \& Heisel, 1958; Leibowitz \& Judisch, 1967) found that the Ponzo illusion increased with age, whereas Pressey and his associates found that it decreased with age (Pressey, 1974; Pressey \& Wilson, 1978). Quina and Pollack (1972), on the other hand, found that the assimilative portion of the Ponzo illusion declined with age whereas the portion that exhibited contrast increased with age. The latter result is in complete agreement with the generalization offered by Pressey (1974) that assimilative illusions will decline and contrast illusions will increase during the early years. One has to assume that Jordan and Randall are referring to the data obtained by Quina and Pollack, who found contrast to occur in the line that was most distant from the apex. My own analysis of the target used by Quina and Pollack indicates that if the center of attention is located at the fixation point and if contrast is mediated through the interactive field, then contrast is exactly what would be expected and, since contrast is assumed to increase with age, the theory remains intact. 
Just as puzzling is the claim by Jordan and Randall that assimilation theory cannot explain the effects of wedge degradation on the Ponzo illusion. Degradation alters the saliency of the contextual stimuli and, as such, both the size of the optimal attentive and optimal interactive field could change. There is no way to deduce from the theory exactly how contrast of the contextual line operates, but the fact that the theory could be extended to incorporate brightness or degradation without major amendment seems obvious. ${ }^{4}$

The problem of how perceptual contrast is mediated is an important one. Can a simple experiment be designed to distinguish between the Jordan-Randall and the PresseyWilson proposals? The following might suffice. Measure the parallel-lines illusion with a single comparison line that is located below the standard line but close enough to it so that both lines can be apprehended simultaneously. Locate a longer contextual line either above or below the standard line but at a constant distance from the standard (see Pressey \& Murray, 1976). Systematically vary the size of the contextual line and measure the illusion. If Jordan and Randall are correct, then contrast should appear with shorter contextual lines when those lines are above the standard than when they are below the standard. If Pressey and Wilson (1980) are correct, there either should be no difference or contrast should appear with shorter contextual lines when those lines are below the standard line. 5

Just such a study has been carried out by Wilson (1981). He found no contrast under any of the conditions in which it would be expected on the basis of past research. Moreover, he has failed to find unequivocal evidence for contrast in the Baldwin illusion (Wilson \& Pressey, in press). This finding is supported by Pressey and Smith (1986), who also failed to find strong evidence for contrast. Thus, at present, the major concern for us is not to establish how contrast is mediated, but rather to determine whether or not contrast can be reliably demonstrated in a visual illusion task.

\section{REFERENCES}

COREn, S., \& Girgus, J. S. (1978). Seeing is deceiving: The psychology of visual illusions. Hillsdale, NJ: Erlbaum.

Farquhar, M., \& Leibowitz, H. W. (1971). The magnitude of the Ponzo illusion as a function of age for large and small stimulus configurations. Psychonomic Science, 25, 97-99.

JoRDAN, K., \& RANDALL, J. (1987). The effects of framing ratio and oblique length on Ponzo illusion magnitude. Perception \& Psychophysics, 41, 435-439.

Kersten, B., Sarris, V., \& Kolbert, J. (1987). Predicted gap effects for the Mueller-Lyer figure: A test of two quantitative models. Unpublished manuscript.

Leibowitz, H. W., \& Heisel, M. A. (1958). L'evolution de l'illusion de Ponzo en fonction de l'age. Archives de Psychologie, 36, 328-331.

Leibowitz, H. W., \& Judisch, J. A. (1967). The relation between age and the Ponzo illusion. American Joumal of Psychology, 80, 105-109.

Pressey, A. W. (1974). Age changes in the Ponzo and filled-space illusions. Perception \& Psychophysics, 15, 315-319.

Pressey, A. W. (1982). The parallel lines illusion as a measure of cognitive style (Report). Winnipeg: Manitoba Mental Health Research Foundation.
Pressey, A. W., Butchard, N., \& Scrivner, L. (1971). Assimilation theory and the Ponzo illusion: Quantitative predictions. Canadian Joumal of Psychology, 25, 486-497.

Pressey, A. W., \& Murray, R. (1976). Further developments in the assimilation theory of geometric illusions: The adjacency principle. Perception \& Psychophysics, 19, 536-544.

Pressey, A. W., \& SMITH, N. E. (1986). The effects of location, orientation, and cumulation of boxes in the Baldwin illusion. Perception \& Psychophysics, 40, 344-350.

Pressey, A. W., \& Wilson, A. E. (1978). Another look at age changes in geometric illusions. Bulletin of the Psychonomic Society, 12, 333-336.

Pressey, A. W., \& Wilson, A. E. (1980). Assimilation theory and the Baldwin illusion. Italian Journal of Psychology, 7, 65-73.

Quina, K., \& Pollack, R. H. (1972). Effects of test line position and age on the magnitude of the Ponzo illusion. Perception \& Psychophysics, 12, 253-256.

WILSON, A. E. (1981). Contrast and assimilation in illusions of extent. Unpublished doctoral dissertation, University of Manitoba, Winnipeg, Canada.

Wilson, A. E., \& Pressey, A. W. (in press). Contrast and assimilation in the Baldwin illusion. Perceptual \& Motor Skills.

\section{NOTES}

1. The original decision to choose contour interaction instead of attentional processes to mediate contrast was not based on empirical data, since no such data were available. Instead, the choice was made because of the quantitative version of the theory. It seemed that contrast effects could appear because the contour fell within the interactive field and could disappear because it fell outside either the interactive or the attentional field. Thus, no new estimates of free parameters were needed to accommodate the phenomenon of contrast. However, if contrast were mediated through the attentional field, the effect would increase indefinitely unless a second edge to the attentional field was included. A quantitative estimate of this second edge, in the absence of any data, involved little more than guesswork.

2. The operational definition of the center of the attentional field is the midpoint between the two most extreme elements that are to be judged, as set by the instructions of the task (Pressey \& Wilson, 1980). The minimum attentional field (i.e., the field that is logically necessary to postulate for the task to be carried out at all) is the radius of a circle that just encompasses those (most distal) elements. The optimum attentional field is the size that provides the best fit to population trends, and the maximum attentional field is the size that is necessary to postulate in order to estimate the optimum size. In simulations that have been carried out on the parallel lines, the Müller-Lyer, the Ponzo, and the Baldwin illusions (Pressey \& Murray, 1976; Pressey \& Wilson, 1980), the optimum size was approximately 1.5 times the minimum size and the maximum was roughly 2.0 times the minimum.

3. Bernd Kersten, at Frankfurt University (Kersten, Sarris, \& Kolbert, 1987), also uses Jordan and Randall's method for determining the attentional field.

4. Research in our laboratory (Pressey, 1982) suggests that increasing the brightness of the contextual features increases the amount of distortion in the parallel lines display but it does so only when the context is at the edge of the display. In other words, from the perspective of assimilation theory, the brighter context increased the probability that attention would be directed to it, but brightness did not alter contour interaction effects (i.e., interactive field size).

5. In order for contrast to occur, the contextual line must fall both within the contrast area of the interactive field and within the attentive field. If the contour fell outside the attentional field before it appeared in the contrast region of the interactive field, then no contrast would be exhibited.
(Manuscript received July 20, 1987; accepted for publication July 22, 1987.) 